\title{
Unveiling the effects of doping small nickel clusters with a sulfur impurity
}

\author{
Abdelaziz Chikhaoui, Mohamed Ziane, \\ Slimane Tazibt, Said Bouarab • Andrés Vega
}

Received: date / Revised version: date

\begin{abstract}
Small free-standing Ni clusters have been widely investigated during the last decade, but not many of their derived chalcogenides, despite their interest in technology and the new prospects that the nanoscale may open. The present work uncovers the effects of the S-doping on the structural, electronic and magnetic properties of $\mathrm{Ni}_{n}, n=1-10$ clusters. Density functional theoretic calculations within the generalized gradient approximation for the exchange and correlation were conducted to explore the structural, electronic, and magnetic properties of the resulting $\mathrm{Ni}_{n} \mathrm{~S}$ chalcogenide nanoparticles. The sulfur impurity is always adsorbed on the 3-fold holow sites available on the nickel host, in qualitative agreement with recent results of $\mathrm{S}$ adsorption on $\mathrm{Ni}(111)$ surfaces. S-doping tends to enlarge the average $\mathrm{Ni}-\mathrm{Ni}$ inter-atomic distance but enhaces the thermodinamical stability of $\mathrm{Ni}$ clusters. It also increases the vertical ionization energy and electron affinity. However, S-doping has a small effect on the magnetism of small Ni clusters. According to the spin-dependent HOMO-LUMO gap, most of these clusters are good candidates as molecular junctions for spin filtering at low bias voltage.
\end{abstract}

Keywords Ab initio calculations - Doping - Electronic properties · Magnetic properties · Nickel · Sulfur

\section{Introduction}

The chalcogenides are an important class of materials from the technological viewpoint due to their significant electrical, optical and chemical characteristics. The applications of chalcogenide materials include a variety of chalcogenide glasses, infra-red sensors, solar energy conversion and window layer $(1 ; 2 ; 3 ; 4 ; 5 ; 6)$.

A. Chikhaoui, Mohamed Ziane, Slimane Tazibt, Said Bouarab

Laboratoire de Physique et Chimie Quantique, Faculté des Sciences, Université Mouloud Mammeri de Tizi-Ouzou, B.P. Nº 17 RP, 15000 Tizi-Ouzou, Algeria

E-mail: aziz_chikhawi@yahoo.fr

Andrés Vega

Departamento de Física Teórica, Atómica y Óptica, Universidad de Valladolid, Paseo Belén 7, E-47011 Valladolid, Spain. 
Among chalcogenide nanomaterials, nickel sulfide NiS presents potential applications in photoconduction (7). In general, sulfur compounds such as transition metal sulfides have important applications in superconduction $(8 ; 9)$, biochemical systems $(10 ; 11)$ and catalysis $(12 ; 13 ; 14)$. As thin films and at the nanoscale, the chalcogenides as it happens with most materials, present a wide range of new properties inherent to the quantum confinement, which deserve to be explored by the Scientific Community. The main characteristic of nanomaterials comes from the sensitivity of their electronic properties with their morphologies, size and composition, that can be completely different from their mesoscopic and macroscopic counterparts, thus offering new prospects for developing a wide range of new applications.

Small free-standing Ni clusters have been widely investigated from different theoretical approaches $(22 ; 23 ; 24 ; 25 ; 26 ; 27 ; 29 ; 30 ; 31 ; 28 ; 32 ; 33 ; 34 ; 35 ; 36 ; 37$; $38 ; 39 ; 40 ; 41 ; 42 ; 43 ; 44 ; 45 ; 46 ; 47 ; 48 ; 49 ; 50)$ and experimentally $(55 ; 56)$ during nearly two decades. However, no studies have been carried out so far regarding the effects of doping small nickel clusters with a light impurity. Oxygen and sulfur impurities are particularly interesting in this context, due to their abundance, their different electronegativity as compared with $\mathrm{Ni}$, and the relevance of nickel oxide and chalcogenides. By doping the pure $\mathrm{Ni}_{n}$ clusters with an electronegative element like sulfur, local charge transfer is expected which could induce a partial ionic contribution to the local bonding in the system. The aim of the present work is to perform a systematic theoretical study of the evolution of the structural, electronic, and magnetic properties of small Ni clusters when doped with a single sulfur inpurity. We performed density functional theoretic calculations of $\mathrm{Ni}_{n} \mathrm{~S}$ clusters in the size range of $n=1-10$. We determined the most stable atomic arrangements, the absolute and relative stabilities, the electronic structure and magnetic properties, and electronic indicators such as ionization potentials and electron affinities.

A description of our theoretical approach is done in the next section. The results are presented in section III in which we first present the structural properties and then we discuss the stability and electronic properties. The main conclusions are summarized at the end.

\section{Computational method}

Calculations were conducted within the density functional theory, using a plane wave basis set and the Projected Augmented Wave (PAW) approach for the core interactions, as implemented in the VASP code (15). We considered the $3 d^{8} 4 s^{2}$ valence configuration for $\mathrm{Ni}$, and $3 s^{2} 3 p^{4}$ for $\mathrm{S}$. A plane wave energy cutoff of 300 $\mathrm{eV}$ was taken. The exchange and correlation effects were treated in the Generalized Gradient Approximation (GGA) by using the Perdew-Burke-Ernzerhof functional (16). A cubic supercell with lateral size of $15 \AA$ was employed for all the calculations. Only the $\Gamma$ point was taken for integrations in the Brillouin zone. The cluster geometry was optimized, without symmetry constraints, until the force on each atom was less than $0.001 \mathrm{eV} / \AA$, and the total energy was converged to $10^{-6}$ $\mathrm{eV}$. For charged clusters, corrections to the total energy were included by considering the full dipole moment in all directions. The corrections were calculated as the energy difference between a monopole, dipole, or quadrupole in the current supercell and the same monopole, dipole or quadrupole placed in a vacuum. Fur- 
thermore, the quadrupole corrections originating from the expectation value of $r^{2}$ were also taken into account.

The lowest-energy structures of pure $\mathrm{Ni}_{n}$ clusters were obtained by considering various initial configurations with different symmetries. Most of the inputs used were taken from previous published works. For doped $\mathrm{Ni}_{n} \mathrm{~S}$ clusters, we considered various initial atomic arrangements with the $\mathrm{S}$ atom occupying the different sites, and also by adsorbing it on all possible sites of several bare $\mathrm{Ni}_{n}$ low-lying isomers (first, second and sometimes third isomers) calculated beforehand. We checked that the results remain unchanged after small displacements of the atoms around their equilibrium positions and also for higher cut-off energies and even more stringent convergence criteria. The relative stability of different isomers was further checked by performing calculations in different spin states to be sure of the total spin of the putative ground state. Although we did not employed an unbiased structural search, we believe that our sampling should be sufficient due to the small size of the clusters.

With the VASP code, the local charges and spin magnetic moments are computed by projecting the plane-wave components of the eigenstates onto spherical waves inside slightly overlapping atomic spheres of Wigner-Seitz radius. Because this projection depends on the choice of the atomic radius, the sum of the local charges and moments is not necessarily and always identical to the total cluster values. In order to overcome this issue when analyzing the local magnetic moments distribution, we performed the analysis by using Bader's method $(17 ; 18 ; 19)$ which is based on partitioning the cluster into atomic volumes by locating the zero-flux surfaces of the electron density field.

The accuracy of the calculation method is checked by comparing the calculated bond length, vibration frequency, ionization potential and electronic affinity of both $\mathrm{Ni}_{2}$ and $\mathrm{S}_{2}$ dimers, with available experimental data. The results for $\mathrm{Ni}_{2}$, summarized in Table 1, show a fairly good agreement between the calculated and the measured values of the different quantities considered. As for the calculations for $\mathrm{S}_{2}$, the consistency of the results with the experimental data can be found in our previous paper on $\mathrm{Fe}_{n} \mathrm{~S}_{2}$ clusters (21). Moreover, aditional test calculations for hexagonal bulk NiS (NiAs structure) give lattice parameters of 3.45 and 5.20 $\AA$ in good agreement with the measured values of 3.44 and $5.32 \AA$ (20).

\section{Results and discussion}

\subsection{Structural properties}

In order to extract conclusions about the effect of $\mathrm{S}$ doping on the structural and electronic properties, we have first determined the putative ground state of the corresponding pure $\mathrm{Ni}_{n}$ clusters which we also compare with previous results available in the literature. The atomic configurations adopted by the putative minimum energy structure of $\mathrm{Ni}_{n}$ and $\mathrm{Ni}_{n} \mathrm{~S}(n=1-10)$ clusters are displayed in Fig.1 and Fig.2. Only the geometries of the putative ground state is described along the text, except if certain isomers are energetically competitive with the ground state. Information about all the low-lying isomers found in the present work can be obtained from the authors upon request. 
$N i_{n}$ clusters. We note that neutral $\mathrm{Ni}_{n}$ clusters have been the subject of several theoretical studies $(22 ; 23 ; 24 ; 25 ; 26 ; 27 ; 29 ; 30 ; 31 ; 28 ; 32 ; 33 ; 34 ; 35 ; 36 ; 37 ; 39 ; 40$; $41 ; 42 ; 43 ; 44 ; 46 ; 47 ; 48 ; 49 ; 50)$ against which we can benchmark our theoretical approach. Let us note that our group already performed DFT calculations (50) for pure Ni clusters by using the SIESTA code $(51 ; 52)$ with the same approximation for exchange and correlation (GGA-PBE) as in the present work. This method is based on norm-conserving pseudopotentials and linear combinations of atomic orbitals as basis sets for which we used triple- $\zeta$ with double polarization functions. In general, our present results for nickel clusters are similar to those previously obtained with the SIESTA code (50). It exist nevertheless some exceptions like $\mathrm{Ni}_{5}$ for which VASP gives a trigonal bipyramid of $\mathrm{D}_{3 h}$ symmetry and total moment of $4 \mu_{B}$, whereas SIESTA gave a square pyramid of $\mathrm{C}_{4 v}$ symmetry and $6 \mu_{B}$ of total moment. However, we point out that the first isomer of $\mathrm{Ni}_{5}$ with total moment of $6 \mu_{B}$ is found at only $0.03 \mathrm{eV}$ of energy above the putative ground state with total moment of $4 \mu_{B}$. Apart from slight relaxations, our optimized geometries of neutral $\mathrm{Ni}_{n}$ clusters, displayed in figures 1 and 2 , are also consistent with previous results published elswhere, except for few sizes in which a similar scenario as in the previously mentioned case of $\mathrm{Ni}_{5}$ in the SIESTA vs VASP benchmark occurs. Different approaches at the DFT level are known to give rise sometimes to slightly different energetic ordering of the low-lying isomers. It is for this reason that recalculating the pure $\mathrm{Ni}$ clusters at the same level of accuracy as the $\mathrm{S}$ doped clusters is appealing for providing a consistent analysis.

$N i_{n} S$ clusters. We discuss first the general trends. We find that the $\mathrm{S}$ atom tends to occupy a 3 -fold hollow site on the $\mathrm{Ni}_{n}$ host clusters, in qualitative agreement with recent DFT calculations (53) where it was shown that the sulfur adsorption on the $\mathrm{Ni}(100)$ and $\mathrm{Ni}(110)$ surfaces takes preferably place on the most coordinated sites.

The computed average $\mathrm{Ni}-\mathrm{Ni}$ distance (AD) in both $\mathrm{Ni}_{n}$ and $\mathrm{Ni}_{n} \mathrm{~S}$ clusters is plotted as a function of cluster size $n$ in Fig. 3a. In pure nickel clusters, it increases up to $n=5$ and then it keeps nearly constant to stabilize around a value of 2.35 $\AA$. The situation is different in the S-doped clusters, where the Ni-Ni distance increases with respect to that of the pure $\mathrm{Ni}_{n}$ clusters although with a lower slope. Let us now analyze in some detail the structures of the different clusters.

The ground state of $\mathrm{Ni}_{2} \mathrm{~S}$ is an isosceles triangle $\left(\mathrm{C}_{2 v}\right.$ symmetry), with Ni-S and $\mathrm{Ni}-\mathrm{Ni}$ bond lengths of 2.03 and $2.32 \AA$, respectively. Its total magnetic moment of $2 \mu_{B}$ results from a parallel magnetic coupling between the Ni atoms $(2 \times 0.82$ $\left.\mu_{B}\right)$ and the $\mathrm{S}$ atom $\left(0.36 \mu_{B}\right)$. We note that $\mathrm{S}$ doping considerably enhances the $\mathrm{Ni}-\mathrm{Ni}$ bond length, as in the pure $\mathrm{Ni}_{3}\left(\mathrm{D}_{3 h}\right)$ these bond lengths are $2.21 \AA$, and even smaller in $\mathrm{Ni}_{2}(2.09 \AA)$, both pure clusters having also $2 \mu_{B}$.

For $\mathrm{Ni}_{3} \mathrm{~S}$, the putative ground state is a trigonal pyramid of $\mathrm{C}_{s}$ symmetry and total moment of $2 \mu_{B}$, as that of $\mathrm{Ni}_{3}$. The local moments on $\mathrm{Ni}$ atoms are $2 \times 0.64$ and $0.66 \mu_{B}$ whereas the induced moment on $\mathrm{S}$ is very small $0.06 \mu_{B}$. A trigonal pyramid of $\mathrm{C}_{3 v}$ is the first isomer, lying at just $0.02 \mathrm{eV}$ above the ground state. The pure $\mathrm{Ni}_{4}$ is a tetrahedron $\left(\mathrm{D}_{2}\right)$ with total moment of $4 \mu_{B}$. In this case, substitutional doping reduces the total magnetic moment. Doping also enhances the average $\mathrm{Ni}-\mathrm{Ni}$ inter-atomic distance.

$\mathrm{Ni}_{4} \mathrm{~S}$ is a trigonal bipyramid of $\mathrm{C}_{3 v}$ symmetry and $4 \mu_{B}$ of total moment.

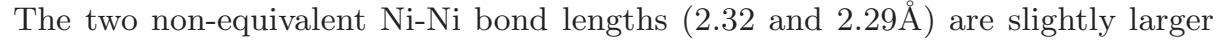


than those of Ni-S bonds $(2.18 \AA)$. The local Ni moments are $3 \times 0.98$ and $0.93 \mu_{B}$ whereas the $\mathrm{S}$ atom is noticeably polarized $\left(0.13 \mu_{B}\right)$. The same atomic arrangement but with a $\mathrm{C}_{s}$ symmetry and the same total moment $\left(4 \mu_{B}\right)$ is found as the first isomer at only $0.01 \mathrm{eV}$ above. The pure $\mathrm{Ni}_{5}$ is a trigonal bipyramid of $\mathrm{D}_{3 h}$ symmetry and total moment of $4 \mu_{B}$, meaning that doping here does not modify the magnetic moment.

The putative lowest-energy structure of $\mathrm{Ni}_{5} \mathrm{~S}$ is a $\mathrm{Ni}_{5}$ distorted trigonal pyramid capped by the $\mathrm{S}$ atom on one of its 3 -fold hollow sites. It has $\mathrm{C}_{s}$ symmetry and a total moment of $4 \mu_{B}$. The pure $\mathrm{Ni}_{6}$ cluster is a square bipyramid of $\mathrm{D}_{4 h}$ symmetry and $8 \mu_{B}$ of total moment resulting from the distribution of the moments of the base atoms $\left(4 \times 1.33 \mu_{B}\right)$ and the apex atoms $\left(2 \times 1.34 \mu_{B}\right)$. This $\mathrm{Ni}_{6}$ structure with $\mathrm{D}_{4 h}$ symmetry is energetically quasi-degenerated $(\Delta \mathrm{E}=0.01 \mathrm{eV})$ with the same atomic arrangement having $\mathrm{C}_{i}$ symmetry. Substitutional doping reduces the total moment and leads to an important atomic rearrangement.

For $\mathrm{Ni}_{6} \mathrm{~S}$, the putative ground state is obtained from the $\mathrm{Ni}_{6}$ square bipyramid where the $\mathrm{S}$ atom is adsorbed on a 3 -fold hollow site giving rise to a $\mathrm{Ni}-\mathrm{Ni}$ bond breaking on the base. It has $\mathrm{C}_{s}$ symmetry and a moment of $6 \mu_{B}$. A similar atomic arrangement but with $\mathrm{C}_{3 v}$ symmetry and the same total magnetic moment as pure $\mathrm{Ni}_{6}\left(8 \mu_{B}\right)$, is found as the first isomer at only $0.04 \mathrm{eV}$ above. The $\mathrm{Ni}_{7}$ cluster is an $\mathrm{Ni}_{6}$ octahedron capped by the seventh $\mathrm{Ni}$ atom on one of its 3 -fold hollow site. It is of $\mathrm{C}_{s}$ symmetry and bear a total moment of $8 \mu_{B}$. As expected, the effect of enlarging the average $\mathrm{Ni}-\mathrm{Ni}$ inter-atomic distance upon $\mathrm{S}$ doping decreases as increasing the size of the Ni cluster.

The putative lowest-energy structure of $\mathrm{Ni}_{7} \mathrm{~S}$ is a distorted pentagonal bipyramid of $\mathrm{C}_{s}$ symmetry where the $\mathrm{S}$ atom caps one of its 3 -fold hollow sites. It bears a total moment of $6 \mu_{B}$. The reduction of the magnetic moment of the host cluster upon $\mathrm{S}$ doping (by $2 \mu_{B}$ ), that takes place for $\mathrm{Ni}_{7} \mathrm{~S}$, as well as for $\mathrm{Ni}_{6} \mathrm{~S}$ is less marked than for the smaller clusters discussed above; for larger clusters, as we will see, this effect dissapears. We will come later to this point when discussing the electronic properties. $\mathrm{Ni}_{8}$ has a bisdisphenoid configuration with $\mathrm{C}_{2 v}$ symmetry and total magnetic moment of $8 \mu_{B}$.

$\mathrm{Ni}_{8} \mathrm{~S}$ is a $\mathrm{Ni}_{6}$ octahedron capped by two $\mathrm{Ni}$ and one $\mathrm{S}$ atoms on 3-fold hollow sites. It has $\mathrm{C}_{s}$ symmetry and total moment of $8 \mu_{B}$. The putative lowest-energy structure of $\mathrm{Ni}_{9}$ is a trigonal prism tricapped of $\mathrm{C}_{2 v}$ symmetry and total moment of $8 \mu_{B}$.

The putative ground state of $\mathrm{Ni}_{9} \mathrm{~S}$ is an octahedron $\mathrm{Ni}_{6}$ capped by three $\mathrm{Ni}$ atoms and the $\mathrm{S}$ atom, on 3 -fold hollow sites. It has the same symmetry $\left(\mathrm{C}_{3 v}\right)$ and same total moment $\left(8 \mu_{B}\right)$ as the putative lowest energy structure of $\mathrm{Ni}_{10}$ where one of the $\mathrm{Ni}$ atoms is replaced by $\mathrm{S}$ (Fig. 2).

Finally, the lowest-energy structure of $\mathrm{Ni}_{10} \mathrm{~S}$ is a distorted pentagonal bipyramid of $\mathrm{C}_{s}$ symmetry and total moment of $8 \mu_{B}$, capped by three $\mathrm{Ni}$ atoms on 3 -fold hollow sites. It was obtained by substiting the tenth atom of the $\mathrm{Ni}_{11}$ cluster by the $\mathrm{S}$ atom (Fig. 2).

\subsection{Stability and electronic properties}

The binding energies (BEs) per atom for both $\mathrm{Ni}_{n}$ and $\mathrm{Ni}_{n} \mathrm{~S}$ clusters $(n=1-10)$ are plotted in Fig. 3c. BE is calculated from the following expressions 


$$
\begin{aligned}
B E\left(N i_{n}\right) & =\quad-\left[E\left(N i_{n}\right)+n E(N i)\right] / n, \\
B E\left(N i_{n} S\right) & =-\left[E\left(N i_{n} S\right)+n E(N i)+E(S)\right] /(n+1) .
\end{aligned}
$$

First, we notice that the general behavior of the BE of pure $\mathrm{Ni}_{n}$ clusters as function of size is consistent with previous theoretical results $(33 ; 34 ; 39 ; 43 ; 46$; $49 ; 50)$. BEs of $\mathrm{Ni}_{n} \mathrm{~S}$ clusters are larger than those of pure $\mathrm{Ni}_{n}$, particularly for the smallest sizes for which the Ni/S rate is larger. As expected, BEs of pure and doped clusters tend to converge to each other as increasing cluster size. This reflects the obvious fact that a single $\mathrm{S}$ atom is averaged with an increasing number of $\mathrm{Ni}$ atoms. For clusters larger than those investigated here, it is expected that the $\mathrm{S}$ atom will continue to occupy preferentially the most coordinated hollow sites, as it occurrs in surfaces (53), with concomitant local relaxations but without affecting the rest of the host cluster. The binding energy indicates that S-doping enhances the thermodinamical stability of the Ni clusters. This binding increase is related to the strong $\mathrm{S}-\mathrm{Ni}$ bonding, reflected in the kind of adsorption site (the S-atom is bonded with three $\mathrm{Ni}$ atoms in a 3 -fold hollow site). As we will see later, the strong $\mathrm{S}-\mathrm{Ni}$ bond is favoured by a partial ionic contribution due to charge transfer from $\mathrm{Ni}$ to $\mathrm{S}$ as a consequence of the different electronegativities of the two elements ( $\mathrm{S}$ is more electronegative than $\mathrm{Ni}$ ).

A further evidence of the strong $\mathrm{S}-\mathrm{Ni}$ bonding is provided by the single-atom fragmentation energies. We considered two single-atom fragmentation channels of $\mathrm{Ni}_{n} \mathrm{~S}$, involving one $\mathrm{Ni}$ atom or the $\mathrm{S}$ atom. These quantities, plotted in Figure $4 \mathrm{a}$ are defined in terms of total energies as follows:

$$
\begin{aligned}
\Delta_{S} & =E\left[N i_{n}\right]+E[S]-E\left[N i_{n} S\right], \\
\Delta_{N i} & =E\left[N i_{n-1} S\right]+E[N i]-E\left[N i_{n} S\right] .
\end{aligned}
$$

One can see from Figure 4 a that $\Delta_{S}$ is larger than $\Delta_{N i}$, for any size $n$, showing that the desulfurization (removing the $\mathrm{S}$ atom) of $\mathrm{Ni}_{n} \mathrm{~S}$ systems requires more energy than extracting the less bonded $\mathrm{Ni}$ atom of the cluster. In other words, it is easier to dissociate $\mathrm{Ni}$ than $\mathrm{S}$ atom from the $\mathrm{Ni}_{n} \mathrm{~S}$ system which suggests that the Ni-S bonding is stronger than the Ni-Ni one. The lowest Ni fragmentation energy corresponds to the extraction of one $\mathrm{Ni}$ atom from $\mathrm{Ni}_{4} \mathrm{~S}$ and the largest desulfurization energy corresponds to the removing the $\mathrm{S}$ atom from $\mathrm{Ni}_{3} \mathrm{~S}$ and $\mathrm{Ni}_{4} \mathrm{~S}$. The maximum difference $(2.77 \mathrm{eV})$ between the two energies is obtained for $\mathrm{Ni}_{4} \mathrm{~S}$. One can connect the low energy required to dissociate one $\mathrm{Ni}$ atom from the $\mathrm{Ni}_{4} \mathrm{~S}$ cluster to the enlargement of the Ni-Ni inter-atomic distances which takes place upon $\mathrm{S}$ doping of $\mathrm{Ni}_{4}$ (Fig. 3a). Besides, the apex $\mathrm{Ni}$ atom in the trigonal bipyramid of $\mathrm{Ni}_{4} \mathrm{~S}$ is the less bounded atom due to the repulsion with its Ni neighboring atoms, induced by the charge transfer to the $\mathrm{S}$ atom (Fig. 4b).

In order to discuss the relative stability of a cluster of a given size $n$ with respect to its neighboring sizes, we computed the second energy difference, defined as $\Delta_{2} E(n)=E(n+1)+E(n-1)-2 E(n)$. This quantity is plotted in Fig. $3 \mathrm{~b}$. For S-doped clusters, the most remarkable feature is the noticeable peak at $n=3$ indicating the high stability of $\mathrm{Ni}_{3} \mathrm{~S}$ for which the completion of the 3 -fold bonding of $\mathrm{S}$ with Ni takes place. Notice that this correlates with the largest desulfurization 
energy corresponding to the remove of the $\mathrm{S}$ atom from $\mathrm{Ni}_{3} \mathrm{~S}$. For pure Ni clusters, $\mathrm{Ni}_{6}$ stands out as particularly stable. As we will see later, these relative stabilities are also reflected in electronic properties like the ionization potential and the electronic affinity.

The vertical ionization potential (VIP) and the vertical electronic affinity (VEA) are important quantities that can be used to characterize the global reactivity of the $\mathrm{Ni}_{n} \mathrm{~S}$ clusters by means of conceptual DFT indicators (54). The VIP and VEA for both the pure and doped nickel clusters are plotted in Fig. 5, as a function of cluster size $n$, where we included the calculated values obtained for the pure $\mathrm{Ni}_{n}$ clusters as well as experimental data reported for them $(55 ; 56)$. The VIP(VEA) of $\mathrm{Ni}_{n} \mathrm{~S}$ clusters is calculated as the energy difference between the neutral clusters and the cationic (anionic) counterparts with the structure of the ground state neutral cluster. Except the result of $\mathrm{Ni}$ atom, for which the difference between the experimental and the calculated values reaches $\sim 0.68 \mathrm{eV}$, the calculated VIPs for $\mathrm{Ni}_{n}$ clusters follow the experimental data (55) rather well as a function of cluster size. This gives further support to our putative ground state structures. Unfortunately, experimental data concerning VIP and VEA of S-doped nickel clusters are not available so far. However, one can bring out some trends concerning the effect of S-doping on VIP of $\mathrm{Ni}_{n} \mathrm{~S}$ by comparing their calculated values with those of the pure $\mathrm{Ni}_{n}$ clusters. From Figure 5, one can see that $\mathrm{S}$ doping produces in general a slight increase of the VIP, except for $n=2$ and 6 . We also note that the VIPs decrease as a function of size in a more monotonic fashion for the S-doped Ni clusters than for the pure ones. As for the calculated values of VEAs for $\mathrm{Ni}_{n}$ clusters, they agree also rather well with the experimental data, except for $n=3$ for which the difference in the absolute values amounts to $0.28 \mathrm{eV}$. The VEA of pure Ni clusters increases quite lineary with $n$ between $n=2$ and 9 and then decreases slightly for $n=10$. The VEA increases upon S-doping by about $11 \%$. It follows almost the same slope as of pure nickel clusters as a function of cluster size $n$. The enhancements of both VEA and VIP of S-doped clusters with respect to those of pure $\mathrm{Ni}_{n}$ are consistent with the higher binding energy of the S-doped clusters as compared with the pure ones (Fig. 3c).

From VIP and VEA one can evaluate the electronegativity (the negative of the electronic chemical potential $\mu$ ):

$$
\chi=-\mu=\frac{1}{2}(V I E+V E A)
$$

and the chemical hardness (or fundamental gap, except for a constant factor):

$$
\eta=E_{g a p}=\frac{1}{2}(V I E-V E A)
$$

These quantities are plotted in Fig. 5 as a function of the cluster size $n$ for the pure and doped nickel clusters. Both $\chi$ and $\eta$ of $\mathrm{Ni}_{n}$ clusters display qualitatively the same behaviour as the ionization energy as function of the size $n$. They decrease between $n=2$ and 4 and then increase up to $n=6$ after which it decreases again up to $n=10$. Thus, both quantities present a local maximum at $n=6$ and a local minimum at $n=4$ which correlates with the relative high stability of $\mathrm{Ni}_{6}$ clusters and the low stability of $\mathrm{Ni}_{4}$ already observed through the second difference in the energy (Figure 3b). The electronegativity of S-doped Ni clusters is equal or higher 
than that of the pure ones. The chemical hardness $\eta$ does not change much, and the most noticeable change occurs for $n=4$.

Let us discuss now the magnetic behavior of the pure and S-doped nickel clusters. The total magnetic moment as a function of cluster size $n$ of both sets of clusters is plotted in Fig. 6a. We have also plotted the magnetic moments per atom (Figure 6b) and included, for the sake of comparison, the available experimental data for pure $\mathrm{Ni}_{n}$ clusters (57). Our values are smaller than the experimental ones, as it is also the case for almost all ab initio calculations on nickel clusters $(33 ; 40 ; 42 ; 47 ; 49)$. Those discrepancies between theory and experiment remain an open question, although the problem may arise from the additional orbital moment contributions or isomerization effects as suggested by some authors $(38 ; 45)$.

The S-doping of $\mathrm{Ni}_{n}$ clusters affects $\mathrm{Ni}_{6} \mathrm{~S}$ and $\mathrm{Ni}_{7} \mathrm{~S}$ by reducing their total moment from $8 \mu_{B}$ in $\mathrm{Ni}_{6}$ and $\mathrm{Ni}_{7}$ to $6 \mu_{B}$. We note that for both $\mathrm{Ni}_{6} \mathrm{~S}$ and $\mathrm{Ni}_{7} \mathrm{~S}$ clusters, the magnetic state with $8 \mu_{B}$ is only $\sim 0.03 \mathrm{eV}$ higher in energy than the ground state with $6 \mu_{B}$. This small effect of S-doping on the total spin polarization of $\mathrm{Ni}_{n}$ clusters is consistent with the weak hybridization between the $\mathrm{S}$ and $\mathrm{Ni}$ orbitals. To analyze this with two examples, we compare the density of states (DOS) of $\mathrm{Ni}_{5} \mathrm{~S}\left(\mathrm{Ni}_{6} \mathrm{~S}\right)$ with that of $\mathrm{Ni}_{5}\left(\mathrm{Ni}_{6}\right)$. [Figures 7 and 8]. In both cases, the hybridization is weak and involves molecular orbitals far from the Fermi level. For $\mathrm{Ni}_{5} \mathrm{~S}$ the geometry of the host $\mathrm{Ni}_{5}$ cluster is essentially preserved (Fig. 1), and no significant rearrangement of both spin-up and spin-down states takes place upon S-doping, the total moment being preserved (Fig. 7). In $\mathrm{Ni}_{6} \mathrm{~S}$, however, we observe an important electronic states rearrangement for both spin components upon $\mathrm{S}$ doping (Fig. 8), associated to a structural change. The spectrum rearrangement is accompanied by a spin flip from the majority to minority spin states, giving rise to a decrease of $2 \mu_{B}$ upon doping.

Finally, we plot in Figure 9, as a function of cluster size, the spin-up and spin-down HOMO-LUMO gaps of $\mathrm{Ni}_{n} \mathrm{~S}$ in their putative lowest-energy structures. The HOMO-LUMO gap of each spin channel is a key quantity for spin-dependent electronic transport at low voltages. The S-doped clusters with $n \leq 4$ and $n=8$ 10, have relatively large HOMO-LUMO gaps for spin-up states $(\sim 2.38$ to 1.20 $\mathrm{eV}$ ) whereas, except for $n=3$, spin-down states have small gaps ( $\sim 0.60$ to 0.04 $\mathrm{eV}$ ). The situation is somewhat different for pure $\mathrm{Ni}_{n}$ clusters where the largest HOMO-LUMO gaps for spin-up states are obtained for $n=2-3$ and for sizes larger than $n=5 . \mathrm{Ni}_{6}\left(\mathrm{Ni}_{4}\right)$ displays the largest(lowest) gap $2.46(0.23 \mathrm{eV})$ which reflects the high(low) stability of this cluster (Figure $3 \mathrm{~b}$ ). One can note that the total gap of $\mathrm{Ni}_{4}$ is the smallest one as reflected in its low stability from the calculated second differences of energy for pure $\mathrm{Ni}_{n}$ clusters (Figure $3 \mathrm{~b}$ ). The gaps corresponding to spin-down states are relatively small, and nearly zero for $n>4$, indicating that these clusters are good candidates as molecular junctions for spin filtering at low bias voltage.

However, the absolute value of the HOMO-LUMO gap has to be taken with care, since it is not a well defined quantity in DFT that tends to underestimate it due to the lack of electronic correlations. Hybrid functionals such as BLYP, B3LYP, B3PW91, HSE06, among others, are designed to better describe part of the exchange and/or correlation effects and, thus, give better absolute values for the HOMO-LUMO gaps than functionals like PBE that otherwise are built from physical grounds and work well for a wide spectrum of properties. To the best of our knowledge only one theoretical work using B3LYP functional (61) was devoted 
to nickel sulfide clusters $(\mathrm{NiS})_{n}, n=3-5$. In order to estimate the discrepancy between the HOMO-LUMO value obtained with PBE and B3LYP, we calculated at the PBE level the ring structure proposed for $(\mathrm{NiS})_{3}$ in this reference. The PBE HOMO-LUMO gap $(0.50 \mathrm{eV})$ underestimates the B3LYP one $(1.04 \mathrm{eV})$ in about $50 \%$. Other quantities such as ionization potential (IP) and electron affinity (EA) and derived quantities are expected to be less affected since they are obtained as energy differences between ground states calculated at the DFT level, so that they are well defined indicators within the DFT as oppose to the HOMO-LUMO gap. This is confirmed by the calculated VIPs and VEAs for $\mathrm{Ni}_{n}$ clusters which follow the experimental data rather well as a function of cluster size, as indicated in Figure 5. For the ring structure proposed for $(\mathrm{NiS})_{3}$ in reference (61), our calculated values of VIP and VEA, 5.65 and $3.5 \mathrm{eV}$ respectively, are within 13 and $18 \%$ of those of B3LYP, 5 and $4 \mathrm{eV}$ respectively (61).

\section{Conclusions}

The main conclusions of our DFT-GGA study of S-doped $\mathrm{Ni}_{n} \mathrm{~S}$ clusters $(n=1-10)$ can be summarized as follows.

(i) The S atom tends to occupy a 3-fold hollow site, in agreement with DFT calculations (53) of sulfur adsorption on Ni surfaces, which was shown to take place preferentially on the most coordinated sites. A further evidence of the strong S-Ni bonding is provided by the single-atom fragmentation energies: a Ni atom can be extracted from $\mathrm{Ni}_{n} \mathrm{~S}$ with a lower energy cost that the $\mathrm{S}$ atom.

(ii) S-doping enhances the thermodynamical stability of the Ni clusters. The increase of binding energy upon $\mathrm{S}$ doping is related to the strong S-Ni bonding, also favoured by a partial ionic contribution due to charge transfer from Ni to $\mathrm{S}$. According to the second differences in energy, $\mathrm{Ni}_{3} \mathrm{~S}$ is particularly stable with respect to the neighboring sizes. All atoms of this cluster are involved in the 3fold bonding, and it has the largest desulfurization energy among the investigated clusters.

(iii) S-doping only affects the magnetic moment of $\mathrm{Ni}_{6} \mathrm{~S}$ and $\mathrm{Ni}_{7} \mathrm{~S}$ by reducing their total moment from $8 \mu_{B}$ in $\mathrm{Ni}_{6}$ and $\mathrm{Ni}_{7}$ to $6 \mu_{B}$. The spin-polarized densities of states show a weak hybridization between the $\mathrm{S}$ and $\mathrm{Ni}$ orbitals, but strong enough structural changes upon doping, in few cases, to produce an electronic states rearrangement consistent with a change of total moment.

(iv) S-doped clusters with $n \leq 4$ and $n=8$-10, have relatively large HOMOLUMO gaps for spin-up states. However, the gaps corresponding to spin-down states are small, and nearly zero for $n>4$, indicating that most of these clusters are good candidates as molecular junctions for spin filtering at low bias voltage.

\section{Aknowledgements}

This study was funded by the Algerian Ministry of Higher Education and Scientific Research via the project CNEPRU B00L02UN150120130013, and by the Junta de Castilla y Len (Spain) (Project VA124G18) 


\section{References}

1. Tikhomirov V K, Asatryan K, Galstian T V, Vallee R, Seddon A B 2003 Philos Mag Lett 83:117-124

2. Jain P K, Deepika K S, Saxena N S 2009 Philos Mag 89:641-650

3. Zogg H, Arnold M 2006 Opto Electron Rev 14:33-36

4. Ruxandra V 1997 J Mater Sci Lett 16:1833-1835

5. Li K, Wee A T S, Lin J, Tan KL, Zhou L, Li S F Y, Feng Z C, Chou H C, Kamra S, Rohatgi A 1997 J Mater Sci Mater Electron 8: 125-132

6. Hartley A, Irvine S J C 2000 J Mater Sci Mater Electron 11:569-573

7. Mane R S, Lokhande C D 2000 Mater Chem Phys 65:1-31

8. Köckerling M, Johrendt D, Finckh E W 1998 J Am Chem Soc 120:12297

9. Chevrel R, Hirrien M, Sergent M 1986 Polyhedron 5:87

10. Stiefel E I, Matsumoto K 1996 ACS Sym Ser 653:2

11. Krebs B, Henkel G 1991 Angew Chem Int Ed Engl 30:769

12. Harris S, Chianelli R R 1984 J Catal 86:400

13. Prins R, de Beer V H G, Somorjai G A 1989 Catal Rev Sci Eng 31:1

14. Paskach T J, Schrader G L, McCarley R E 2002 J Catal 211:285

15. Kresse G, Hafner J 1993 Phys Rev B 47:558

16. Perdew J P,Burke K, Ernzerhoh M 1996 Phys Rev Lett 77:3865-3868

17. Henkelman G, Arnaldsson A, Jónsson H 2006 Comput Mater Sci 36:354-360.

18. Sanville E, Kenny S D, Smith R, Henkelman G 2007 J Comput Chem 28:899908

19. Tang W, Sanville E, Henkelman G 2009 J Phys Condens Matter 21:084204-7

20. Vaidya N, Indian J 1976 Pure Appl Phys 14:600

21. Tazibt S, Chikhaoui A, Bouarab S, Vega A 2017 J Phys Chem A 121:3768-3780

22. Mlynarski P, Salahub D R 1991 J Chem Phys 95:6050

23. Jellinek J, Garzón I L 1991 Z Phys D 20:239

24. Stave M S, DePristo A E 1992 J Chem Phys 20:3386

25. Garzón I L, Jellinek J, J. in Physics and Chemistry of Fine Systems, From Clusters to Crystals, edited by Jena, P.; Khanna, S. N.; Rao, B. K.; Kluwer Academic, Dordrecht, 1992; Vol. I, p. 405.

26. López M J ,Jellinek J 1994 Phys Rev A 50:1445

27. Menon M, Connolly J, Lathiotakis N, Andriotis A 1994 Phys Rev B 50:8903

28. Reuse F, Khanna S N 1995 Chem Phys Lett 234:77

29. Lathiotakis N N, Andriotis A N, Menon M, Connolly J 1996 J Chem Phys 104:992

30. Bouarab S, Vega A, Lopéz M J, Iñiguez M P, Alonso J 1996 Phys Rev B 55:13279

31. Castro M, Jamorski C, Salahub D R 1997 Chem Phys Lett 271:133

32. Nayak S K, Rao B K, Jena P 1997 J Phys Chem A 101:1072

33. Reddy B V, Nayak S K, Khanna S N, Rao B K, Jena P 1998 J Phys Chem A 102:1748

34. Doye J P K, Wales D J 1998 New J Chem 22:733

35. Curotto E, Matro A, Freeman D L, Doll J D 1998 J Chem Phys 108:729

36. Michaelian K, Rend N, Garz I L 1999 Phys Rev B 60:2000

37. Xiang Y, Sun D Y, Gong X G 2000 J Phys Chem A 104:2746

38. Khanna S N, Beltran M, Jena P 2001 Phys Rev B 64:235419

39. Michelini M C, Pis Diez R, Jubert A H 2001 Int J Quan Chem 85:22 
40. Duan H M, Gong X G, Zheng Q Q, Lin H Q 2001 J Appl Phys 89:7308

41. Luo C L 2002 Materials Science and Engineering 10:13

42. Ashman C, Khanna S N, Pederson M R 2003 Chem Phys Lett 368:257

43. Grigoryan V G, Spingborg M 2004 Phys Rev B 70:205415

44. Xie Z, Ma Q M, Liu Y, Li Y C 2005 Phys Lett A 342:459

45. Aguilera-Granja J M, Montejano-Carrizales R A, Guirado-Lpez R A 2006 Phys Rev B 73:115422

46. Deshpandre M D, Roy S, Kanhere D G 2007 Phys Rev B 76:195423

47. Yao Y H, Gu X, Ji M, Gong X G, Wang D S 2007 Phys Lett A 360:629

48. Lee B, Lee G W 2007 J Chem Phys 127:164316

49. Lu Q L, Luo Q Q, Chen L L, Wan J G 2011 Eur Phys J D 61:389

50. Chikhaoui A, Haddab K, Bouarab S, Vega A 2011 J Phys Chem A 115:1399714005

51. Soler J M, Artacho E, Gale J D, Garca A, Junquera J, Ordejon P, SanchezPortal D 2002 J Phys Condens Matter 14:2745

52. Ordejon P, Artacho E, Soler J M 1996 Phys Rev B 53:R10441

53. Kandaskalov D, Monceau D, Mijoule C, Damien 2013 Surf. Sci. 617:15-21

54. Geerlings P, De Proft F, Langenaeker W 2003 Chem Rev 103:1793-1873

55. Knickelbein M B, Yang S, Riley S J 1990 J Chem Phys 93:94

56. Rienstra-Kiracofe J C, Tschumper G S, Schaefer H F III, Nandi S, Ellison G B 2002 Chem Rev 102:231

57. Apsel S E, Emmert J W, Deng J, Bloomfield L A 1996 Phys Rev Lett 76:1441

58. Pinegar J C, Langenberg J D, Arrington C A, Spain E M, Morse M D 1995 Chem Phys 102:666

59. Wang H, Haouari H, Craig R, Lombardi J R, Lindsay D M 1996 J Chem Phys 104:3420

60. Ahmed F, Nixon E R 1979 J Chem Phys 71:3547

61. Nagarajan V, Chandiramouli R, Sriram S, Gopinath P 2014 J. Nanostruct Chem 4:87 
Table 1 Calculated values of bond length $r$, vibrational frequency $\omega$, ionization potential IP and electronic affinity $\mathrm{EA}$ for $\mathrm{Ni}_{2}$ dimer, compared to experimental data.

\begin{tabular}{lcccc}
\hline $\mathrm{Ni}_{2}$ Dimer & $\mathrm{r}(\AA)$ & $\omega\left(\mathrm{cm}^{-1}\right)$ & $\mathrm{IP}(\mathrm{eV})$ & $\mathrm{EA}(\mathrm{eV})$ \\
\hline This work & 2.09 & 320 & 7.91 & 1.0 \\
Exp. works & $(2.15)^{a}$ & $259.2 \pm 3.0^{b}$ & $7.65^{c}$ & $0.93^{d}$ \\
& $381^{e}$ & & \\
\hline
\end{tabular}

${ }^{a}(58),{ }^{b}(59),{ }^{c}(55),{ }^{d}(56),{ }^{e}(60)$

Table 2 Interatomic distances $\mathrm{r}_{i j}(\AA)$ in $\mathrm{Ni}_{n}, n=7-11$ clusters according to the labels of the different atoms of the figure 2

\begin{tabular}{|c|c|c|c|c|}
\hline $\mathrm{Ni}_{7}$ & $\mathrm{Ni}_{8}$ & $\mathrm{Ni}_{9}$ & $\mathrm{Ni}_{10}$ & $\mathrm{Ni}_{11}$ \\
\hline $\begin{array}{l}\mathrm{r}_{12}=\mathrm{r}_{25}=\mathrm{r}_{51}=2.32 \\
\mathrm{r}_{34}=\mathrm{r}_{46}=\mathrm{r}_{63}=2.32 \\
\mathrm{r}_{14}=\mathrm{r}_{45}=\mathrm{r}_{35}=2.35 \\
\mathrm{r}_{32}=\mathrm{r}_{26}=\mathrm{r}_{16}=2.35 \\
\mathrm{r}_{17}=\mathrm{r}_{27}=\mathrm{r}_{57}=2.32\end{array}$ & $\begin{array}{l}\mathrm{rr}_{16}=\mathrm{r}_{26}=2.36 \\
\mathrm{r}_{17}=\mathrm{r}_{27}=2.35 \\
\mathrm{r}_{14}=\mathrm{r}_{23}=2.37 \\
\mathrm{r}_{36}=\mathrm{r}_{46}=2.28 \\
\mathrm{r}_{35}=\mathrm{r}_{45}=2.37 \\
\mathrm{r}_{38}=\mathrm{r}_{48}=2.36 \\
\mathrm{r}_{37}=\mathrm{r}_{47}=2.29 \\
\mathrm{r}_{58}=2.32 \\
\mathrm{r}_{12}=2.34\end{array}$ & $\begin{array}{l}\mathrm{r}_{14}=\mathrm{r}_{16}=\mathrm{r}_{17}=\mathrm{r}_{19}=2.37 \\
\mathrm{r}_{26}=\mathrm{r}_{29}=\mathrm{r}_{34}=\mathrm{r}_{37}=2.37 \\
\mathrm{r}_{25}=\mathrm{r}_{28}=\mathrm{r}_{35}=\mathrm{r}_{38}=2.36 \\
\mathrm{r}_{45}=\mathrm{r}_{65}=\mathrm{r}_{78}=\mathrm{r}_{98}=2.35 \\
\mathrm{r}_{46}=\mathrm{r}_{79}=2.36 \\
\mathrm{r}_{47}=\mathrm{r}_{69}=2.24 \\
\mathrm{r}_{85}=2.32\end{array}$ & $\begin{array}{l}\mathrm{r}_{15}=\mathrm{r}_{35}=\mathrm{r}_{31}=2.41 \\
\mathrm{r}_{26}=\mathrm{r}_{46}=\mathrm{r}_{24}=2.42 \\
\mathrm{r}_{27}=\mathrm{r}_{48}=\mathrm{r}_{69}=2.28 \\
\mathrm{r}_{37}=\mathrm{r}_{57}=\mathrm{r}_{39}=2.28 \\
\mathrm{r}_{19}=\mathrm{r}_{18}=\mathrm{r}_{58}=2.28 \\
\mathrm{r}_{14}=\mathrm{r}_{16}=\mathrm{r}_{32}=2.41 \\
\mathrm{r}_{36}=\mathrm{r}_{52}=\mathrm{r}_{54}=2.41\end{array}$ & $\begin{array}{l}\mathrm{r}_{12}=\mathrm{r}_{13}=2.37 \\
\mathrm{r}_{24}=\mathrm{r}_{35}=2.38 \\
\mathrm{r}_{45}=2.43 \\
\mathrm{r}_{26}=\mathrm{r}_{36}=2.31 \\
\mathrm{r}_{46}=\mathrm{r}_{56}=2.60 \\
\mathrm{r}_{16}=2.33 \\
\mathrm{r}_{27}=\mathrm{r}_{37}=2.38 \\
\mathrm{r}_{47}=\mathrm{r}_{57}=2.42 \\
\mathrm{r}_{17}=2.32 \\
\mathrm{r}_{48}=\mathrm{r}_{58}=2.38 \\
\mathrm{r}_{67}=2.59 \\
\mathrm{r}_{68}=2.31 \\
\mathrm{r}_{411}=\mathrm{r}_{511}=2.28 \\
\mathrm{r}_{711}=2.28 \\
\mathrm{r}_{92}=\mathrm{r}_{103}=2.37 \\
\mathrm{r}_{94}=\mathrm{r}_{105}=2.32 \\
\mathrm{r}_{96}=\mathrm{r}_{106}=2.37 \\
\mathrm{r}_{98}=\mathrm{r}_{108}=2.36\end{array}$ \\
\hline
\end{tabular}


Table 3 Interatomic distances $\mathrm{r}_{i j}(\AA)$ in $\mathrm{Ni}_{n}, n=7-11$ clusters according to the labels of the different atoms of the figure 2

\begin{tabular}{|c|c|c|c|c|}
\hline $\mathrm{Ni}_{6} \mathrm{~S}$ & $\mathrm{Ni}_{7} \mathrm{~S}$ & $\mathrm{Ni}_{8} \mathrm{~S}$ & $\mathrm{Ni}_{9} \mathrm{~S}$ & $\mathrm{Ni}_{10} \mathrm{~S}$ \\
\hline $\begin{array}{l}\mathrm{r}_{13}=\mathrm{r}_{24}=2.29 \\
\mathrm{r}_{36}=\mathrm{r}_{46}=2.33 \\
\mathrm{r}_{15}=\mathrm{r}_{25}=2.27 \\
\mathrm{r}_{3 S}=\mathrm{r}_{4 S}=2.15 \\
\mathrm{r}_{35}=\mathrm{r}_{45}=2.42 \\
\mathrm{r}_{16}=\mathrm{r}_{26}=2.38 \\
\mathrm{r}_{34}=2.15 \\
\mathrm{r}_{65}=2.43 \\
\mathrm{r}_{6 S}=2.24\end{array}$ & $\begin{array}{l}\mathrm{r}_{17}=\mathrm{r}_{27}=2.32 \\
\mathrm{r}_{13}=\mathrm{r}_{24}=2.38 \\
\mathrm{r}_{15}=\mathrm{r}_{25}=2.42 \\
\mathrm{r}_{35}=\mathrm{r}_{45}=2.39 \\
\mathrm{r}_{16}=\mathrm{r}_{26}=2.36 \\
\mathrm{r}_{36}=\mathrm{r}_{46}=2.38 \\
\mathrm{r}_{3 S}=\mathrm{r}_{4 S}=2.13 \\
\mathrm{r}_{34}=2.53 \\
\mathrm{r}_{57}=2.37 \\
\mathrm{r}_{56}=2.47 \\
\mathrm{r}_{56}=2.47 \\
\mathrm{r}_{67}=2.28 \\
\mathrm{r}_{5 S}=2.20\end{array}$ & $\begin{array}{l}\mathrm{r}_{15}=\mathrm{r}_{45}=2.42 \\
\mathrm{r}_{25}=\mathrm{r}_{35}=2.38 \\
\mathrm{r}_{12}=\mathrm{r}_{34}=2.34 \\
\mathrm{r}_{16}=\mathrm{r}_{46}=2.42 \\
\mathrm{r}_{26}=\mathrm{r}_{36}=2.36 \\
\mathrm{r}_{17}=\mathrm{r}_{48}=2.31 \\
\mathrm{r}_{27}=\mathrm{r}_{38}=2.31 \\
\mathrm{r}_{57}=\mathrm{r}_{58}=2.29 \\
\mathrm{r}_{1 S}=\mathrm{r}_{4 S}=2.18 \\
\mathrm{r}_{14}=2.54 \\
\mathrm{r}_{23}=2.35 \\
\mathrm{r}_{6 S}=2.19\end{array}$ & $\begin{array}{l}\mathrm{r}_{15}=\mathrm{r}_{13}=\mathrm{r}_{35}=2.42 \\
\mathrm{r}_{24}=\mathrm{r}_{46}=\mathrm{r}_{62}=2.54 \\
\mathrm{r}_{19}=\mathrm{r}_{39}=\mathrm{r}_{37}=2.28 \\
\mathrm{r}_{75}=\mathrm{r}_{85}=\mathrm{r}_{81}=2.28 \\
\mathrm{r}_{27}=\mathrm{r}_{69}=\mathrm{r}_{48}=2.31 \\
\mathrm{r}_{2 S}=\mathrm{r}_{4 S}=\mathrm{r}_{6 S}=2.16\end{array}$ & $\begin{array}{l}\mathrm{r}_{17}=\mathrm{r}_{27}=2.37 \\
\mathrm{r}_{13}=\mathrm{r}_{24}=2.40 \\
\mathrm{r}_{15}=\mathrm{r}_{25}=2.29 \\
\mathrm{r}_{35}=\mathrm{r}_{45}=2.64 \\
\mathrm{r}_{18}=\mathrm{r}_{29}=2.36 \\
\mathrm{r}_{85}=\mathrm{r}_{95}=2.34 \\
\mathrm{r}_{38}=\mathrm{r}_{49}=2.34 \\
\mathrm{r}_{310}=\mathrm{r}_{410}=2.40 \\
\mathrm{r}_{810}=\mathrm{r}_{910}=2.37 \\
\mathrm{r}_{16}=\mathrm{r}_{26}=2.40 \\
\mathrm{r}_{16}=\mathrm{r}_{26}=2.40 \\
\mathrm{r}_{36}=\mathrm{r}_{46}=2.50 \\
\mathrm{r}_{3 S}=\mathrm{r}_{4 S}=2.16 \\
\mathrm{r}_{34}=2.50 \\
\mathrm{r}_{76}=2.34 \\
\mathrm{r}_{6 S}=2.16 \\
\mathrm{r}_{57}=2.34 \\
\mathrm{r}_{510}=2.29\end{array}$ \\
\hline
\end{tabular}




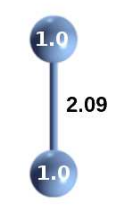

Coov, 2

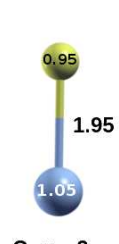

Cov, 2

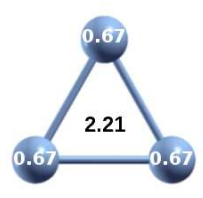

D3h, 2

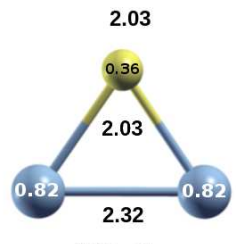

C2v, 2

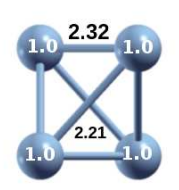

D2, 4

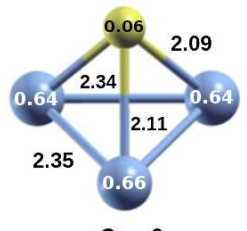

Cs, 2

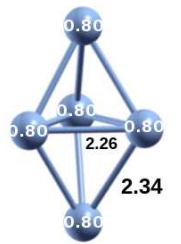

D3h, 4

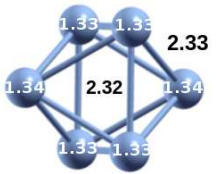

D4h, 8
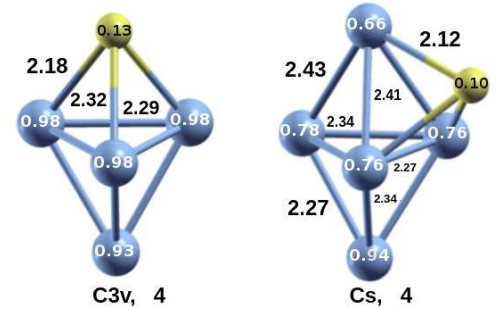

Fig. 1 Putative ground-state structures of $\mathrm{Ni}_{n}, n=2-6$ (first line) and $\mathrm{Ni}_{n} \mathrm{~S}, n=1-5$ (second line) clusters, with their corresponding point-group symmetry and total magnetic moment $\left(\mu_{B}\right)$. Bond lengths are given in $\AA$ and the local spin moments are given (in $\mu_{B}$ ) inside the atomic spheres.

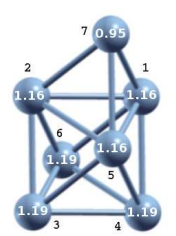

Cs, 8

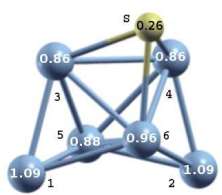

Cs, 6
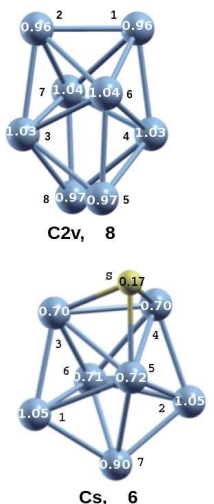
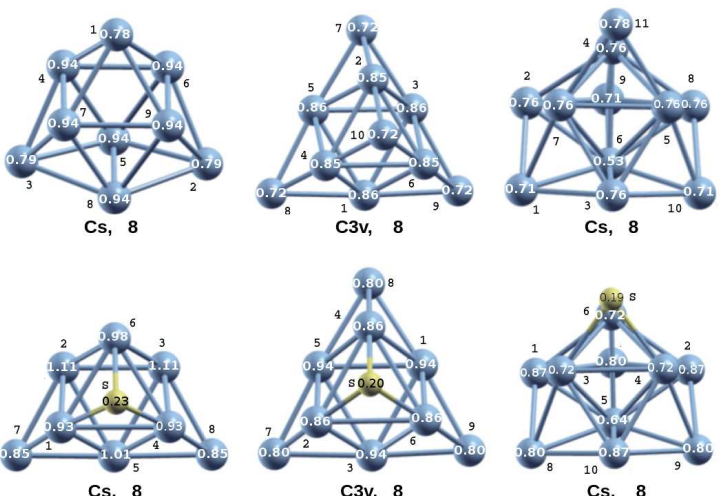

Fig. 2 Putative ground-state structures of $\mathrm{Ni}_{n}(n=7-11)$ and $\mathrm{Ni}_{n} \mathrm{~S}(n=6-10)$ clusters. with their corresponding point-group symmetry and total magnetic moment $\left(\mu_{B}\right)$. The local spin moments are given $\left(\right.$ in $\mu_{B}$ ) inside the atomic spheres whereas the bond lengths are given in table 2 according to the labels of the different atoms. 

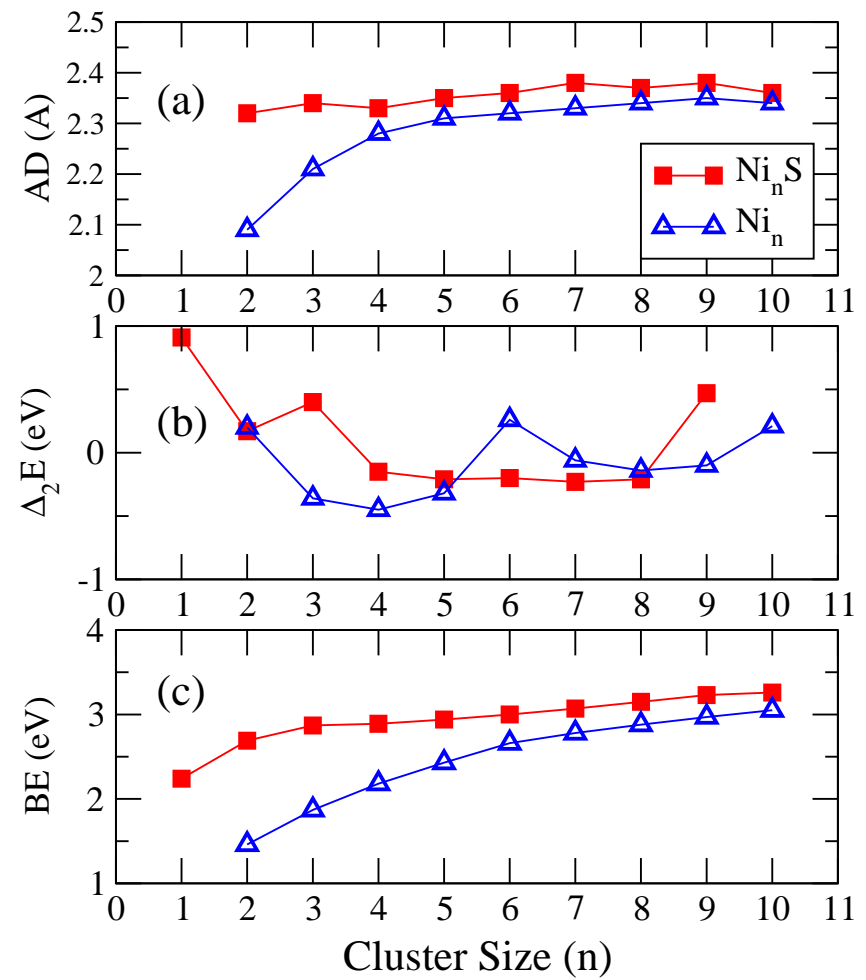

Fig. 3 (a) Calculated average Ni-Ni distance (AD), (b) second energy difference $\left(\Delta_{2} E\right.$ ), and (c) binding energy (BE) as function of cluster size $n=1-10$. 

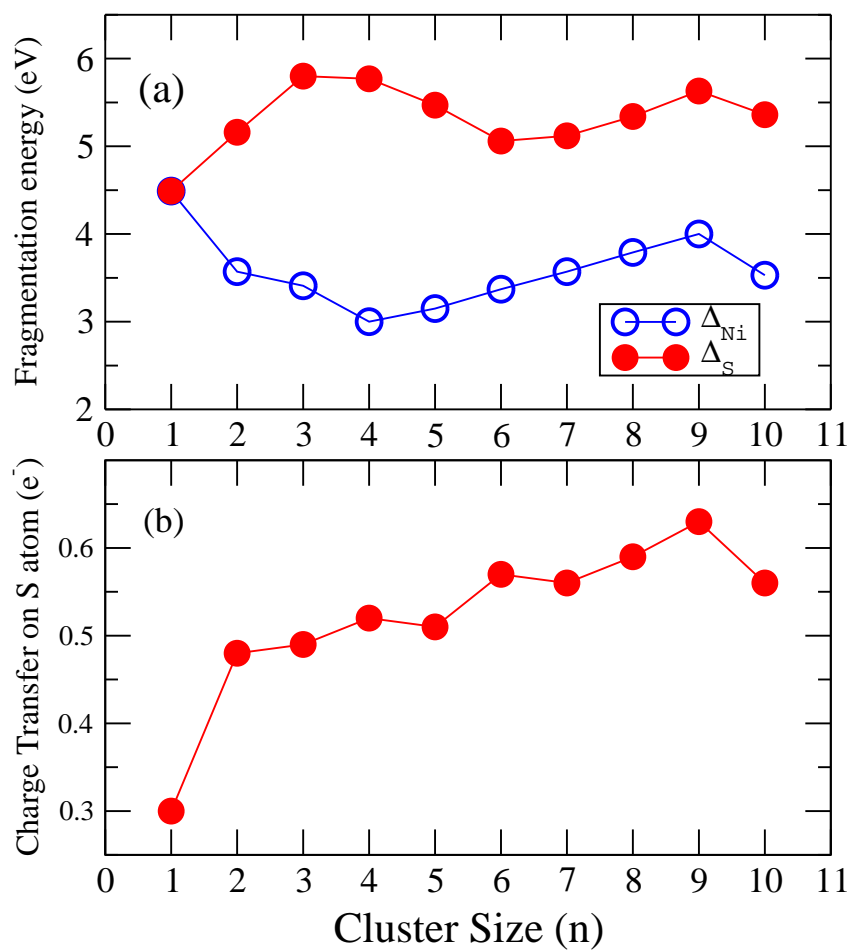

Fig. 4 Fragmentation energies of $\mathrm{Ni}_{n} \mathrm{~S}$ clusters via the loss a $\mathrm{Ni}\left(\Delta_{N i}\right)$ or a $\mathrm{S}\left(\Delta_{S}\right)$ atom, as a function of cluster size $n=1-10$ and calculated charge transfer to the sulfur atom in neutral $\mathrm{Ni}_{n} \mathrm{~S}$ clusters, as function of cluster size $n=1-10$ (b). 

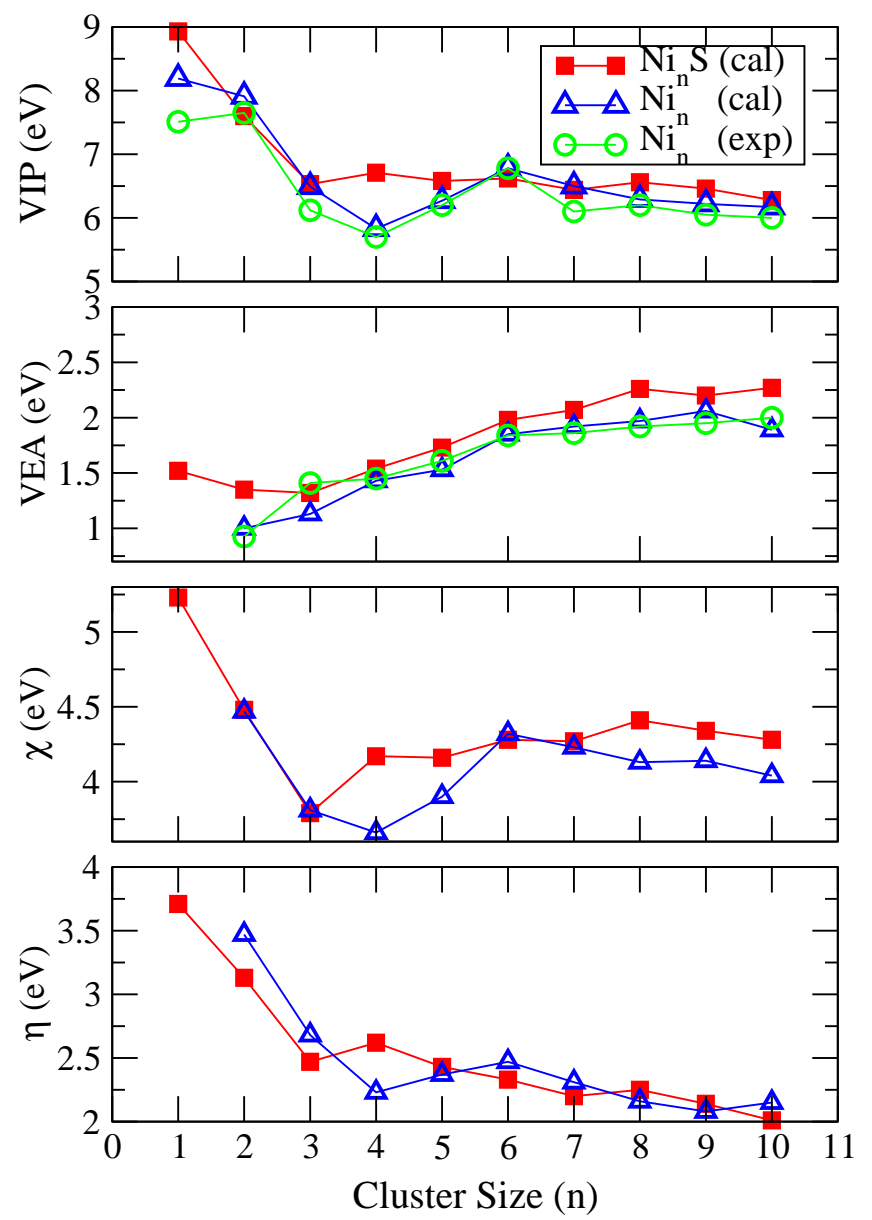

Fig. 5 Vertical ionization potential (VIP), vertical electronic affinity (VEA), electronegativity $(\chi)$ and chemical hardness $(\eta)$ of $\mathrm{Ni}_{n}$ (open triangle) and $\mathrm{Ni}_{n} \mathrm{~S}$ (filled square) as function of the cluster size $n=1-10$. 

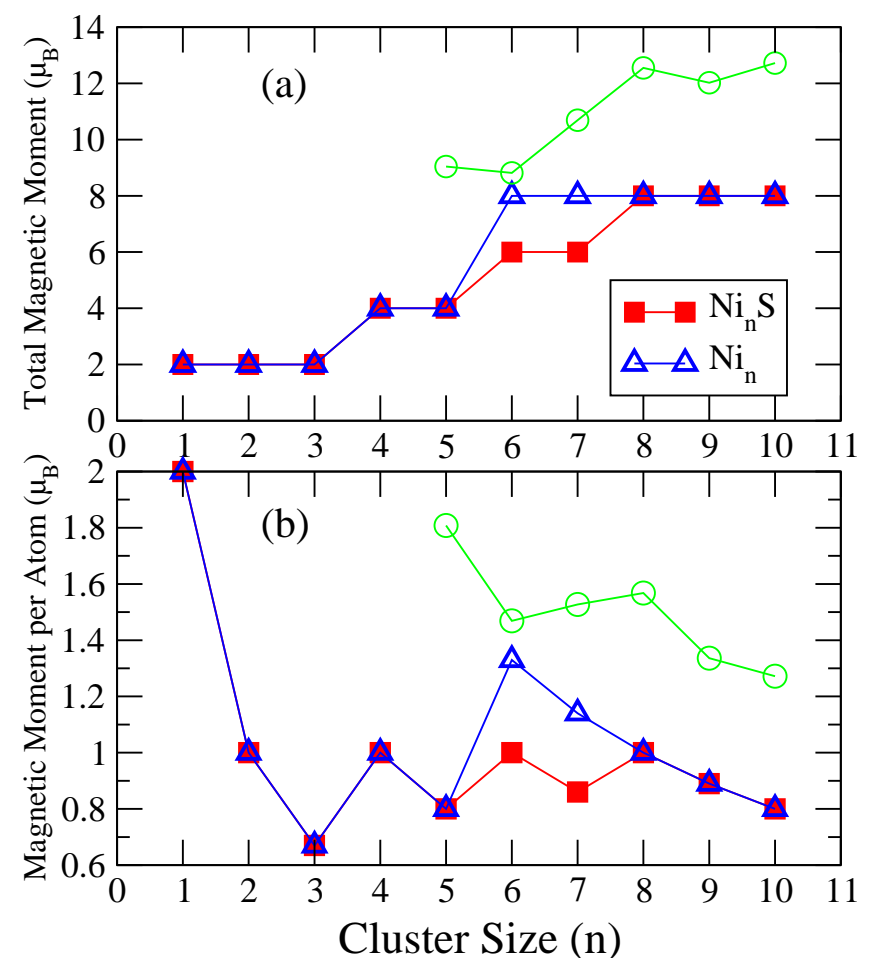

Fig. 6 Total magnetic moments (a) and magnetic moments per atom (b) of $\mathrm{Ni}_{n} \mathrm{~S}$ and $\mathrm{Ni}_{n}$ as a function of cluster size $n=1-10$. 


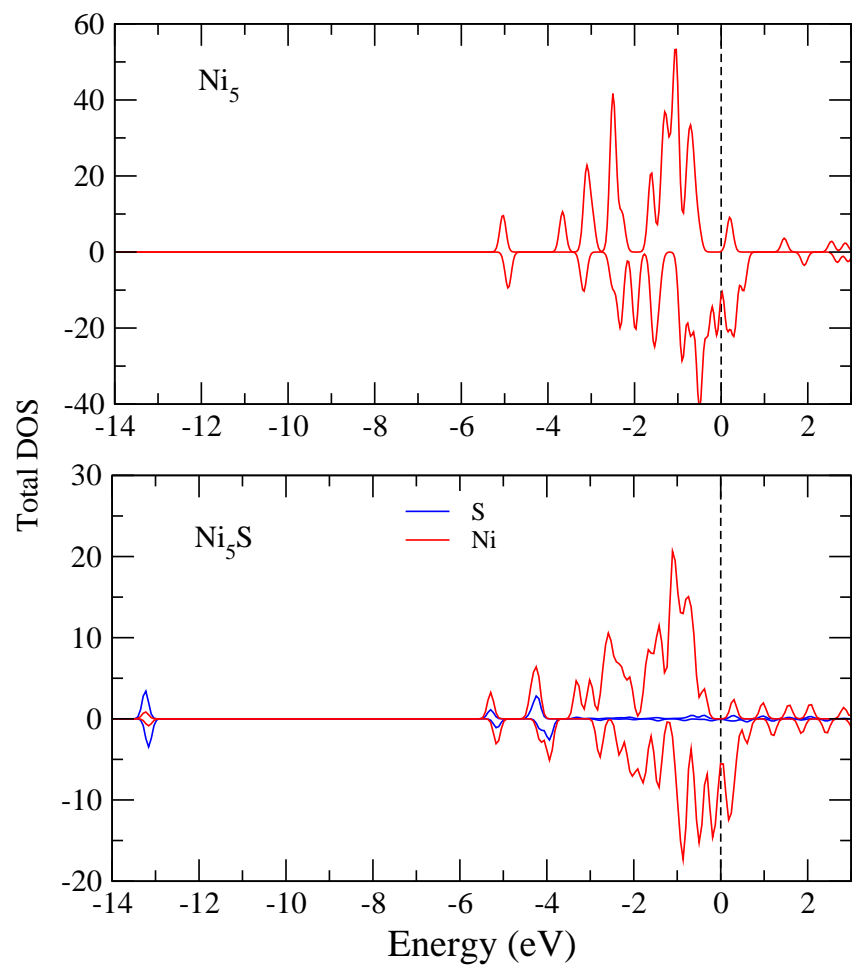

Fig. 7 Total spin polarized density of states projected on the $5 \mathrm{Ni}$ atoms and on the $\mathrm{S}$ atom of the putative ground-state of $\mathrm{Ni}_{5} \mathrm{~S}$ (a), as compared to the total DOS of the host $\mathrm{Ni}_{5}$ (b). The vertical dashed line indicates the Fermi energy. A Gaussian smearing $(0.1 \mathrm{eV})$ of the states has been used in the plot. 


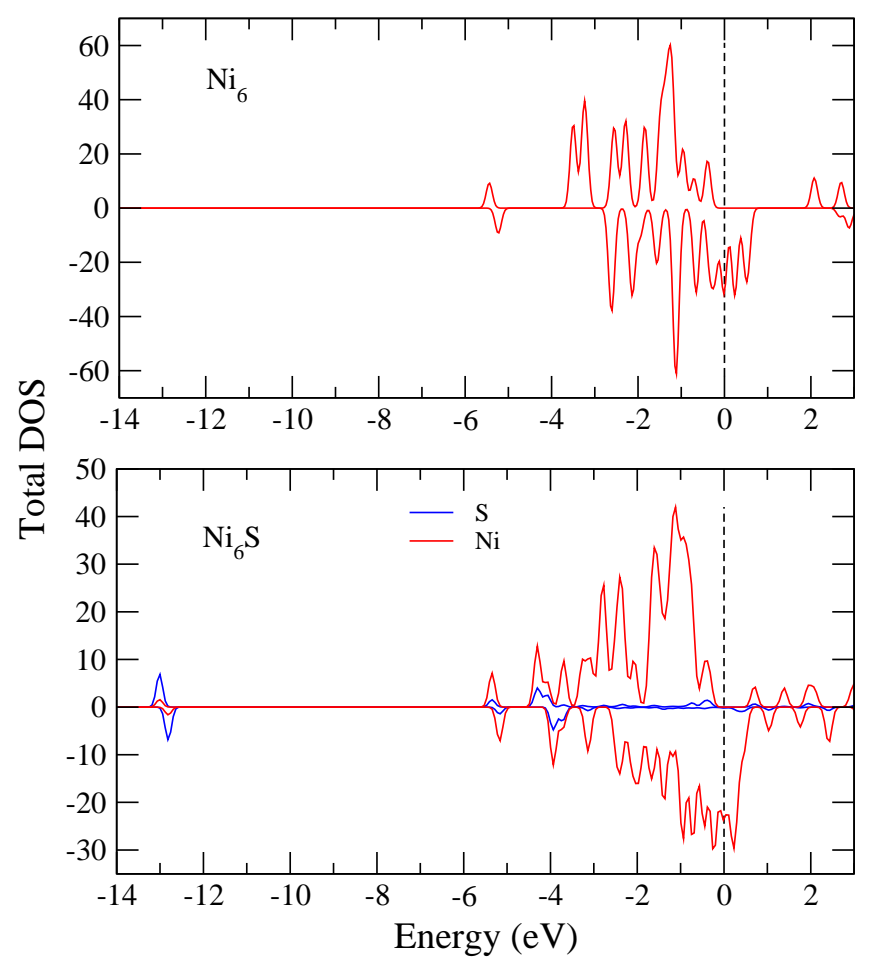

Fig. 8 Total spin polarized density of states projected on the $6 \mathrm{Ni}$ atoms and on the $\mathrm{S}$ atom of the putative ground-state of $\mathrm{Ni}_{6} \mathrm{~S}$ (a), as compared to the total DOS of the host $\mathrm{Ni}_{6}$ (b). The vertical dashed line indicates the Fermi energy. A Gaussian smearing $(0.1 \mathrm{eV})$ of the states has been used in the plot. 


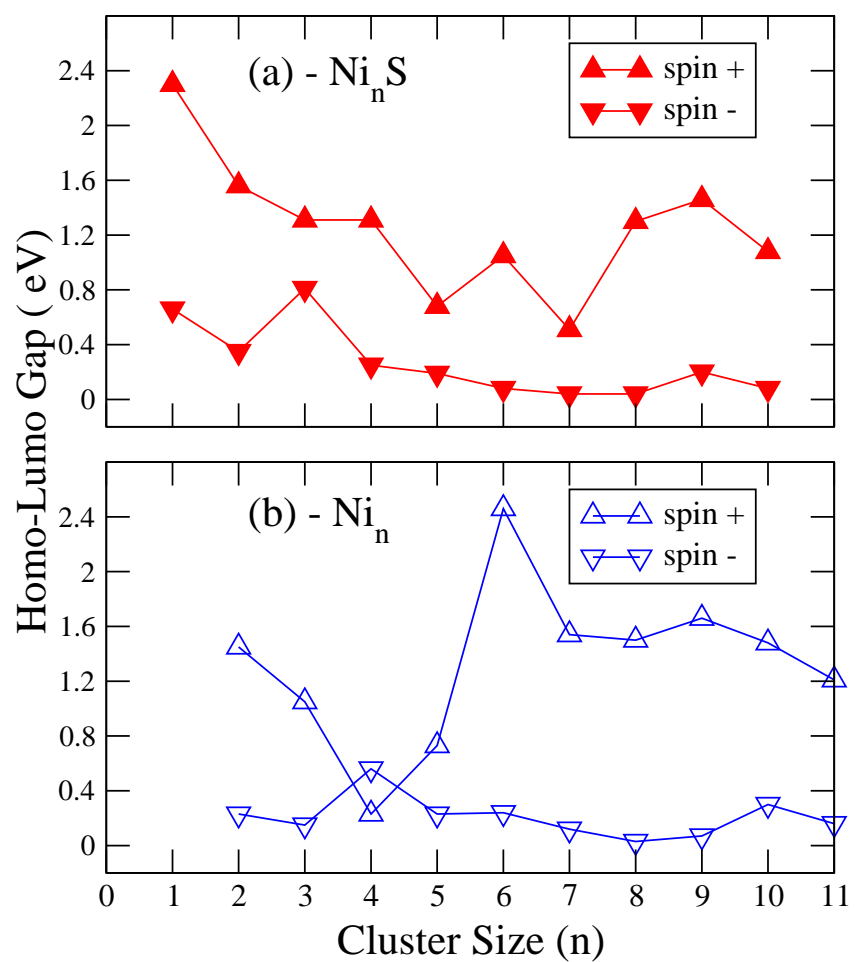

Fig. 9 HOMO-LUMO gap for spin-up and spin-down electrons of $\mathrm{Ni}_{n} \mathrm{~S}$ (a) and $\mathrm{Ni}_{n}$ (b) as function of cluster size $n=1-10$. 